\title{
Haptic Device for Capturing and Simulating Hand Manipulation Rehabilitation
}

\author{
Manuel Ferre, Ignacio Galiana, Raul Wirz and Neil Tuttle
}

\begin{abstract}
This article describes the preliminary development of a haptic setup for capturing and simulating musculoskeletal assessment and manipulation of the hand. A haptic device, called MasterFinger-2, is used for capturing one massage technique and one joint manipulation technique, and also for simulating this manipulation technique that can be used in both assessment and treatment of the hand. First, works developed demonstrate that application of haptic devices enable quantitative characterization of forces and positions used in manipulation of musculoskeletal structures. Secondly, an application for simulation is developed using the MasterFinger-2 to display (both visually and haptically) manipulations of one joint of the hand around three axes. The novel aspects of this approach are the use of a multifinger device for capture, simulation and modeling the movement of a biological joint for haptic simulation across three axes, each with non-linear behavior.
\end{abstract}

Index Terms - Haptic interface, manipulation rehabilitation, hand rehabilitation, multifinger haptic device, multi-contact point, virtual environment.

\section{INTRODUCTION}

Musculoskeletal pain is one of the most common conditions for which people seek medical attention. Treatment to reduce pain and restore normal movement often involve the application of specific forces to the muscles, tendons, ligaments, joints and bones. The assessment and treatment of musculoskeletal dysfunction often involves mobilizing or manipulating joints in specific directions. Forces used in assessing movement of the lumbar [1] and cervical [2] spines have been shown to vary by up to one order of magnitude across practitioners. Similarly forces used in treatment can vary by up to $500 \%$ between practitioners [3,4]. The techniques used in the above studies involved applying a force to one location and measured either the force applied by one finger or the net force applied to the body. Many techniques however are

This work was supported in part by a Grant of Griffith University (Australia) and two Spanish programs of the Ministry of "Ciencia y Tecnología' named 'Juan de la Cierva' and 'Formación de Personal Investigador (FPU)' both focused on training programs for young researchers.

Manuel Ferre, Ignacio Galiana and Raul Wirz are with the Universidad Politécnica de Madrid (UPM), Spain, at the 'Centre for Automation and Robotics (CAR UPM-CSIC)' (email: m.ferre@upm.es, ignacio.galiana@upm.es,r.wirz@upm.es)

Neil Tuttle is with the School of Physiotherapy and Exercise Science at Griffith University, Australia (n.tuttle@griffith.edu.au ) more complex and involve using multiple points of contact. Even a seemingly simple movement such as passively bending one joint in a patient's hand typically involves the clinician using one of their hands to stabilize the proximal segment and the other hand to move the distal segment.

Subtle differences in joint stiffness are thought to be clinically important, but little is known about the objective characteristics of these differences. Early attempts to understand joint mobilization characterized the movement with linear approximations of stiffness [5], but other studies have demonstrated that both the stiffness of the movements [6] and the changes in the stiffness that are related to patient symptoms [7] are complex and non-linear. Perhaps as a result, the interpretation of manual assessment of joint movement remains largely subjective as illustrated by Maher et al [8] who listed over 30 terms used by physiotherapists to describe joint movement with few clear and no objective definitions.

The complexity of movements used to assess and treat joints, and the lack of objective data [9] on either the production or interpretation of the movements may go some way towards explaining the difficulty students have in learning skills for manual assessment and treatment. Recent developments in technology enable not only the measurement of techniques using multiple points of contact, but also the simulation of techniques to improve both the understanding of the movements and the ability to teach students the necessary skills. The soft tissue and joints of the hand provide relatively simple examples of the types of techniques that can be used to assess and treat musculoskeletal dysfunction.

Virtual reality systems incorporating haptic devices are currently being used in a variety of medical environments $[10,11]$. These platforms usually include two key elements: a haptic interface and a graphical simulation of the environment. The vast majority of haptic applications are focused on single contact point interaction between the user and the virtual scenario. A single contact point can be suitable for haptic applications such as palpation or tissues exploration. However, more complex tasks such as grasping or manipulating muscles requires two or more contact points and simulation of tasks using multiple fingers can significantly increase the realism of haptic interactions. During rehabilitation of hand function, some force feedback devices directly exert forces to the finger of the user in order to provide controlled environments to exercise the fingers $[12,13,14]$. The use of virtual reality systems 
including multifinger haptic interfaces have been developed to assist patients' to improve their hand function [15, 16] and this kind of platforms [17] have been shown to be effective in improving coordination [18]. Some examples of these developments are based on exoskeletons [19], gloves $[20,21]$ or other complex multi-point interfaces [22, 23]. To date, however haptic systems have not been used to simulate the types of tasks that a clinician would use in assessing or treating a patient with hand problems.

The aim of this article is to describe the preliminary development of a virtual reality system for simulating musculoskeletal assessment and treatment of the hand, where at least two contact points are required. This setup is based on the MasterFinger-2 [24] device that allows both recording of movements and forces applied by two points of contact during performance of real manipulations; and force feedback during simulations of the recorded scenarios which can then be performed on a virtual hand model [25]. Authors have cooperated at distance in defining and evaluating the manipulation patterns. One author (Neil Tutle) is an experienced physiotherapist and has defined and checked the hand manipulation procedures. Authors from Universidad Politécnica de Madrid (UPM) have customized the haptic device and carried out the manipulation tasks according to procedure defined. Thus, even in this development process, a combination of force and visual data exchanged between remote locations were used to facilitate skill development.

This article is organized as follows: next section describes the two-finger haptic device that has been used in this work, which is called the MasterFinger-2 (MF-2). Some common manipulation hand tasks are described in section three. In section four, capture of gesture, forces and movements by using the haptic device are discussed for two tasks. Section five applies the data from section four and

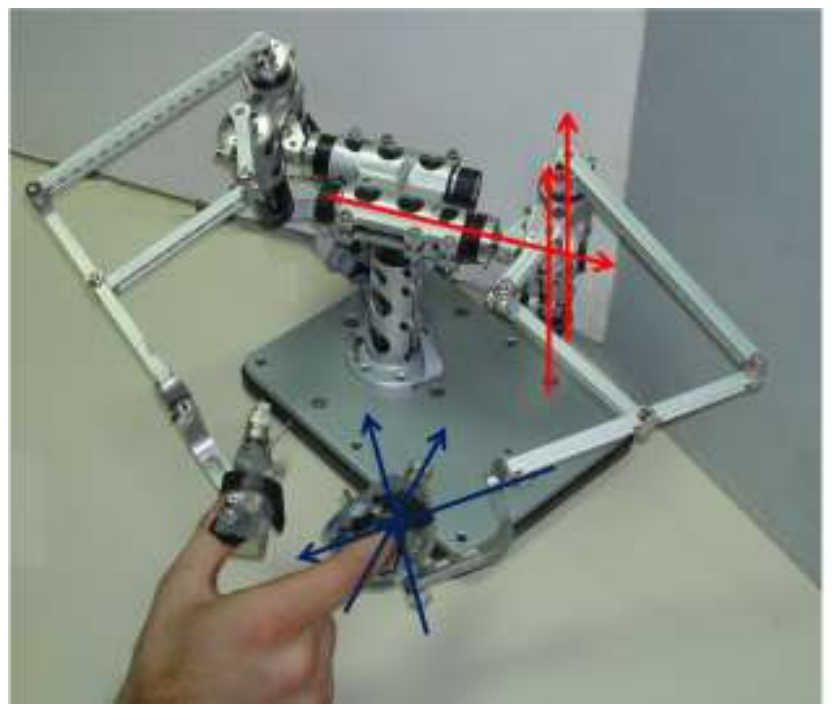

describes the simulation developed for assessing and treating one joint of a finger. Finally, conclusions of the study and potential applications to other human medical manipulations are presented in section six.

\section{HAPTIC DeVICE SET-UP DescriPTION FOR MOdELING HAND MANIPULATION}

A set-up based on the MasterFinger-2 (MF-2) haptic device has been customized for works described in this paper. Fig. 1 shows a view of this haptic interface and a detailed view of the sensorized thimble [26].

The user's index and thumb fingers are inserted into the corresponding thimbles, which are connected to the mechanical structure. As distinct from many haptic devices where either feedback is only provided to one finger or where two points of contact are linked, the mechanical design of the MF-2 is based on a modular configuration where each finger's mechanical structure and electronic components are independent.

\section{A. Multifinger haptic device description}

The two-finger haptic device has 7 actuators and 13 DoFs for movements as shown in Fig.1.a. Each finger has its own mechanical structure with 6 DoFs, the first three DOFs are actuated and allow reflecting forces to the user in any direction and the last three DoFs are only measured and allow the user to achieve any orientation within the workspace. This configuration allows any combination of position and orientation of the thimbles in the device workspace. The three actuators are located close to the base of the device in a serial-parallel configuration that allows reflecting forces to be produced in any direction with minimum inertia. The actuators are linked to a five-bar mechanical structure which in turn is connected to a gimbal with 3 rotational DoFs. The last rotation of the gimbal is

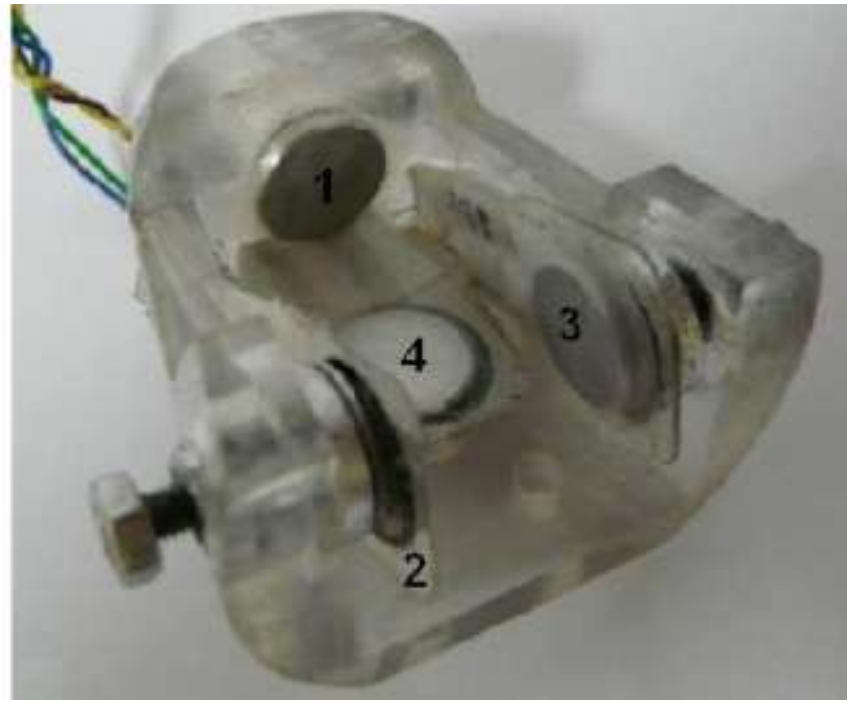

Fig. 1. (a) Two-finger haptic device is shown on the left. Each finger has 6 degrees of freedom for movements. The firsts 3 DoFs are actuated and the last 3 DoFs only measured (arrows at the thimble). On the right, a detail of the thimble is shown. (b) User inserts the index or thumb in the thimble, which have 4 contact sensors that estimate the force applied by the user. 
linked to the thimble. The three gimbal rotational axes intersect in a point close to the user's finger tip. This mechanical configuration allows reflecting forces without the production of torque components.

Mechanical structures of both fingers are linked in the base device and a redundant actuated join is added. This redundant DoF results in a significant increase the size of the workspace, resulting is a workspace approximating the shape of a torus [27]. Finger tip positions are calculated from encoders included in the actuators. Orientations are obtained from three encoders located in the gimbal rotations.

\section{B. Thimble and force sensing resistors}

The thimble shown in Fig 1.b has been designed so that it can be adjusted to fit different size fingers using screws on either side of thimble. In order to measure forces that are both normal and tangential to the finger, the insides of thimbles are fitted with four Flexiforce model A201 (Tekscan Inc.) force sensing resistors (FSRs). Each FSR is mechanically isolated by a metal disk on each side. This 'sandwich' configuration assures a homogeneous distribution of the forces across the contact area and significantly increases the sensor performance. Thimbles containing the FSRs are used to measure the force in each direction exerted by the user during the object manipulation. The accuracy of the thimbles was previously found to be $\pm 1.43 \mathrm{~N}$ in a range from 0 to $20 \mathrm{~N}$ [28], a high precision Force/Torque ATI sensor was used to calibrate and quantify the error.

The signal from the FSR and associated electronic components [29] is filtered and converted to a digital signal by an ADC converter. It is also scaled in order to produce an estimation of the corresponding user component force.

The four FSRs are used for estimating the normal and tangential forces exerted by the user. The normal force is considered to be the net force from the two perpendicular sensors on the end and pad of the finger (numbers 1 and 4 in Fig. 1.b). Tangential forces are estimated via sensors that are placed on both sides of the thimble (numbers 2 and 3 in Fig. 1.b).

\section{CharaCterizATION OF MANiPUlation APPLIED TO HUMAN HAND FOR REHABILITATION}

Manipulation in rehabilitation can have a variety of meanings including fine motor tasks performed by the patient and high velocity, low amplitude thrusts for therapeutic effect. In this work manipulation will be used to refer to relatively slow application of force - typically less than $40 \mathrm{~N} / \mathrm{s}$ - to muscles, tendons, ligaments, joints, bones, or other tissues which has a primary goal of assessment or restoration of normal body motion including the elimination of pain secondary to disturbed biomechanics.

There are perhaps two main divisions of these so-called passive movements. First is massage where the intention is to move or deform soft tissues and the second is mobilization where the intention is to move joint structures. Massage to one location and mobilization of one joint will be used as examples of medical manipulations in this article.

\section{A. Characterization of massage}

There are a wide variety of massage techniques that include various combinations of percussion, compression, stretching and movements parallel to the skin surface.

Massage uses mechanical deformation of the skin, muscle, interstitial fluid and/or fascia to assess or influence their function. In this article, a simple circular massage technique has been used to the web between the thumb and index fingers with minimal compression. This type of technique is of technical interest because the mobility of the skin is not homogenous and varies with direction of force. Similar techniques can be used clinically for assessment as well as treatment for reduction of muscle tone or normalization of tissue texture.

\section{B. Characterization of mobilization}

There are two joints within each finger - the proximal and distal interphalangeal joints - and a metacarpophalangeal (MCP) joint where the finger meets the palm. Each joint can be considered to have three perpendicular axes around which it can be moved during assessment and treatment. These three rotations are referred to as physiological movements because they can be produced actively by the individual and consist of flexion/extension, abduction/adduction and pronation/supination (or axial rotation). The MCP joint of the index finger is capable of all of these movements and is a relatively common source of symptoms so will be used as an example in this study.

The pattern of stiffness in each movement around each axis of each joint is individual and complex. Typically the stiffness of each movement is nonlinear and includes a central region of low stiffness referred to as the lax or neutral zone and a region of higher stiffness near the extremes [30]. Manual assessment of joint function considers both the size of the neutral zone and the behavior of the stiffness near the extremes known as the endfeel. Similarly treatment by passive mobilization can consist of either repeated movements through the neutral zone to modulate pain behavior or oscillations into the area of increased stiffness to increase available range of movement [31].

When assessing or treating physiological movements of the MCP joint, one hand typically stabilizes the metacarpal bone and two fingers of the other hand hold the proximal phalange as close as possible to the joint to apply a force to the bony segment and produce a moment around the joint. This movement typically consists of applying an oscillating force with a frequency from one to perhaps $6 \mathrm{~Hz}$ near the boundary of the joint. Note that in the case of an injured finger, the joint's limit of rotation is before the normal 
finger's range of movement. Practitioners usually select the initial force according to the patient's condition and adjust the force in response to changes that occur in the structure or tissue during the application of the technique.

\section{CAPture of Manipulation Hand Patterns For REHABILITATION}

This section describes the development of a model of soft tissue and MCP joint behaviour using in vivo measurements taken during performance of the massage and mobilization techniques described in the previous section.

To provide reference data, measurements were taken during two techniques that are used in both assessment and treatment: 1) massage of the web between the thumb and index finger, and 2) passive movements of the MCP joint of the index finger. The operator's thumb and index finger were fitted to the thimbles of the MF-2 and the movements were performed with the actuators inactivated. In this way force data could be obtained from the FSRs located in the thimbles, position data from the encoders located in the actuators, and orientation data from the encoders on the axes of the gimbals.

One of the challenging aspects of this project was that the experienced clinician (NT) is in Australia, while the other authors are in Spain. The clinician recorded forces used to perform the techniques and provided descriptions and images. After practice, the other authors sent videos and force traces taken while performing the techniques for feedback. Although less than ideal, this process is not unreasonable as professors currently use video and separate force data [34] in teaching and assessment both when students are on campus and remotely when online.

\section{A. Massage over soft tissue}

For massage, the reference data was obtained while

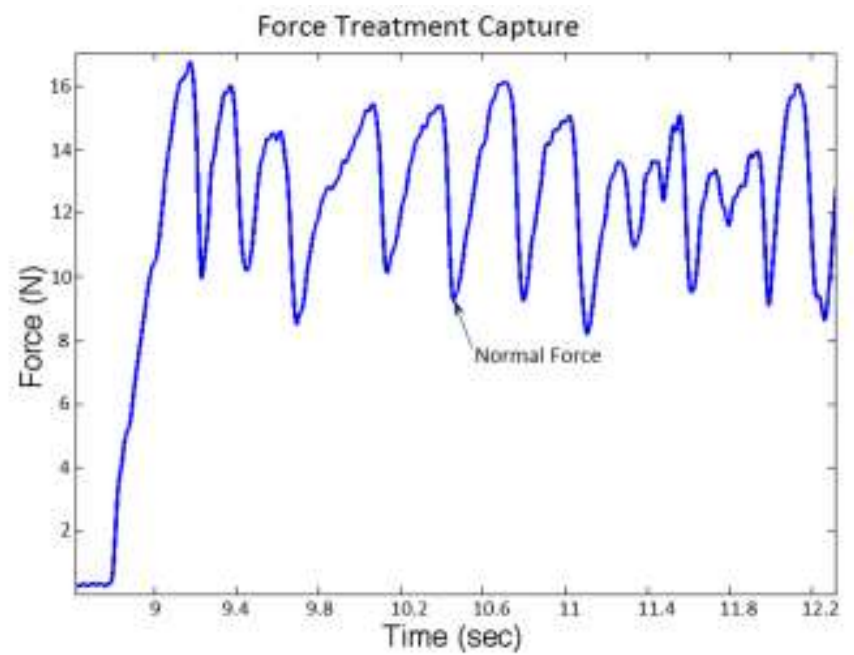

performing circular massage to the web between the thumb and index finger of the subject using the operator's index finger on the subject's palmar surface and the operator's thumb on the subject's dorsal surface. The contact between the operator's thumb and the subject's skin occurred between the two sensors on the midline of the thimble so the output from each of these sensors would include components of forces that were both normal and tangential to the subject's skin. Nonetheless, the force from the two midline sensors will be referred to as normal and the net force from the two lateral sensors as tangential. The measurements from the two lateral sensors also contained the component force used to fix the thimble to the user's finger, however, due to the symmetry of the thimble, this force is the same on the sensor located at both sides of the finger; the net normal and tangential forces can be calculated as the following:

$$
\begin{aligned}
& \mathrm{F}_{\text {tangential }}=\mathrm{F}_{\text {sensor2 }}-\mathrm{F}_{\text {sensor3 }} \\
& \mathrm{F}_{\text {normal }}=\operatorname{sqrt}\left(\mathrm{F}_{\text {sensor } 4}{ }^{2}+\mathrm{F}_{\text {sensor } 1}{ }^{2}\right)
\end{aligned}
$$

The resulting force patterns shown in Fig.2a can be described as:

- Normal forces mostly varied by $\pm 2 \mathrm{~N}$ either side of a fairly constant force of approximately $10 \mathrm{~N}$.

- Lateral forces, measured from the fingertip sides, are similar to a sinusoidal signal including both positive and negative values.

The lateral forces could have been represented by circular patterns, which correspond to frequency of the circle movement performed during the massage. However, since skin and soft tissue are not homogeneous structures and the direction and magnitude of forces will vary during the movement, this would not result in an accurate model. As shown in Fig. 2, only tangential forces on the side of the finger and normal forces were measured with the thimble described in section II.

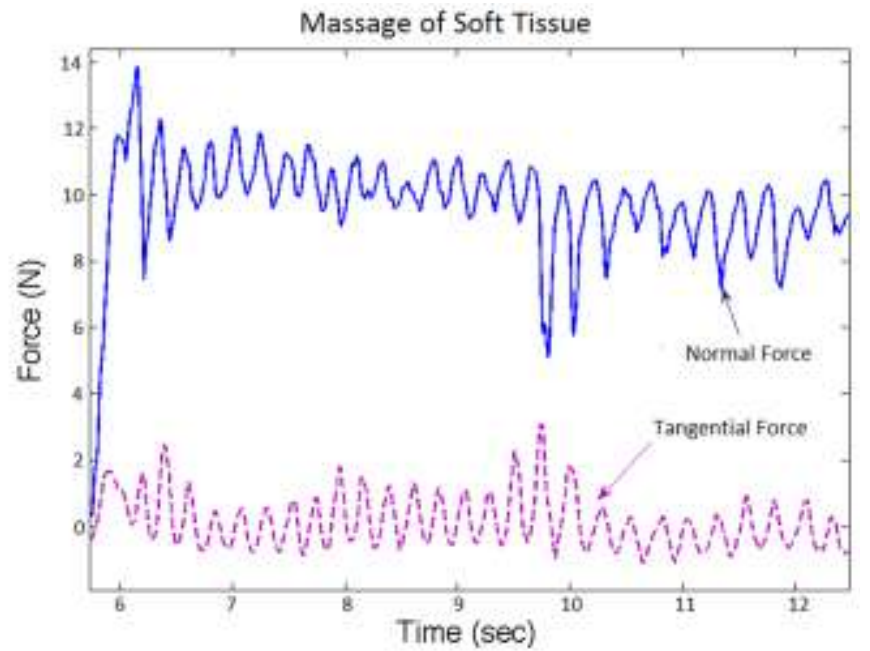

Fig. 2. Force recordings of different hand manipulations. force treatment of the index finger in the Flexion/Extension axis (on the left).and Massage of the soft tissue region between the index and thumb fingers (on the rigth) 

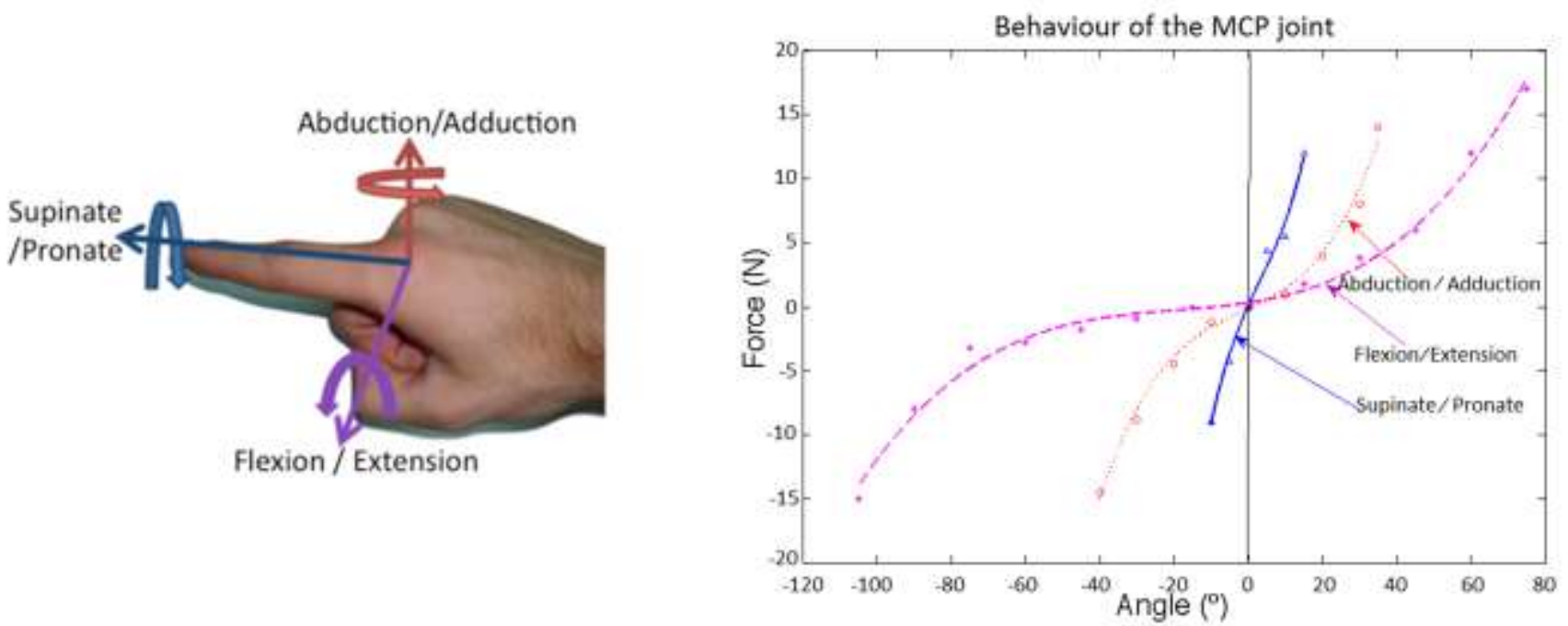

Fig. 3. Data obtained for the metacarpal joint of the index finger (on the rigth). These data are related to the three rotations that can be done in MCP joint as it is shown in the left picture.

\section{B. Mobilization of the MCP joint}

The reference data for stiffness characteristics of rotation around each axis of the MCP joint were calculated from the experimental data and integrated into a virtual model of the hand that can be interrogated using the MF-2 haptic device. In the virtual model, the metacarpal bone will be fixed to ground so the two-finger interface of the MF-2 can be used to manipulate the proximal phalanx of the index finger.

Joint stiffness is likely to vary with rate of movement so the joint was moved at approximately $3 \mathrm{~Hz}$ through a range $(12-16 \mathrm{~N})$ when collecting the reference data as this is near the middle of suggested rates of mobilization used in treatment [31]. Force angle plots for each of the three rotation axes were produced from the force and location data measured during performance of the techniques and are shown in Fig. 3b.

A minimal square interpolation was fitted to the points for each axis of movement to estimate the joint's behavior. The curves demonstrate that the angular limits of the MCP joint are different in the two directions for each axis.

Because a linear interpolation would result in a nonrealistic model, a third order interpolation was used to fit the data. Equations obtained are:

$\mathrm{F}_{\alpha}=1.834 \mathrm{E}-5 \cdot \alpha^{3}+0.0010338 \cdot \alpha^{2}+0.046382 \cdot \alpha+[3]$ 0.59057

$\mathrm{F}_{\beta}=0.00017 \cdot \beta^{3}+0.0012 \cdot \beta^{2}+0.13 \cdot \beta-0.49$

$\mathrm{F}_{\gamma}=0.0014 \cdot \gamma^{3}-0.014 \cdot \gamma^{2}+0.65 \cdot \gamma+0.09$

where $\alpha$ represents the flexion / extension angle, $\beta$ represents adduction / abduction angle, and $\gamma$ represents the pronate / supinate angle as shown in Fig 3.a.

The minimal square error for these polynomial interpolations are $0.49 \mathrm{~N}, 0.3852 \mathrm{~N}$ and $0.0715 \mathrm{~N}$ respectively which are less than the sensorized thimble's error.

The forces were applied at approximately $1 \mathrm{~cm}$. from the MCP joint center of rotation as shown in Fig. 4. Equations 3-5 can be easily converted into torque/angle equations for each axis. These curves were used in next section for modeling the stiffness of the MCP joint of the index finger. Our results were similar to those of a previous in vitro study of flexion/extension stiffness using fresh frozen cadaver's fingers [32]. In addition, a recording of the force and location data was collected with the setup described above as shown in Fig.3.b. at a frequency of approximately $3 \mathrm{~Hz}$. with a force oscillating from 12 to $16 \mathrm{~N}$.

\section{HAPTIC SimUlation OF HUMAN HAND REHABILITATION TASKS}

In this section, an application is described to simulate manual medical manipulations on the MCP joint based on the data gathered in the previous section. This application integrates a two-finger haptic device with a computer model of the human hand that incorporates processes to enable the haptic interaction. This scenario is focused on simulating movement of the MCP joint of the index finger.

It is hoped that the developed application may be useful for comparing techniques of manipulations and for student training. Two factors are crucial for developing a realistic haptic simulation: user's interface and physical engine of the manipulation. The user's interface is based on the MF-2 haptic device and a graphical visual representation of the manipulation scene. On the other hand, the physical engine simulates the interaction among objects (collisions, reaction forces, movements, etc.) according to its dynamical properties.

Physx is a motor engine developed by Nvidia where the dynamics calculations are performed by the Graphics 
Processing Unit (GPU). It is used for implementing the dynamic simulation. To simulate the scenario in real time, a graphic card that is compatible with Physx engine is necessary to ensure the calculations can occur fast enough.

These calculations include collision detection among the different objects; in case of collision, the points that are in contact ( $\mathrm{X}, \mathrm{Y}, \mathrm{Z}$ values) and the penetration between

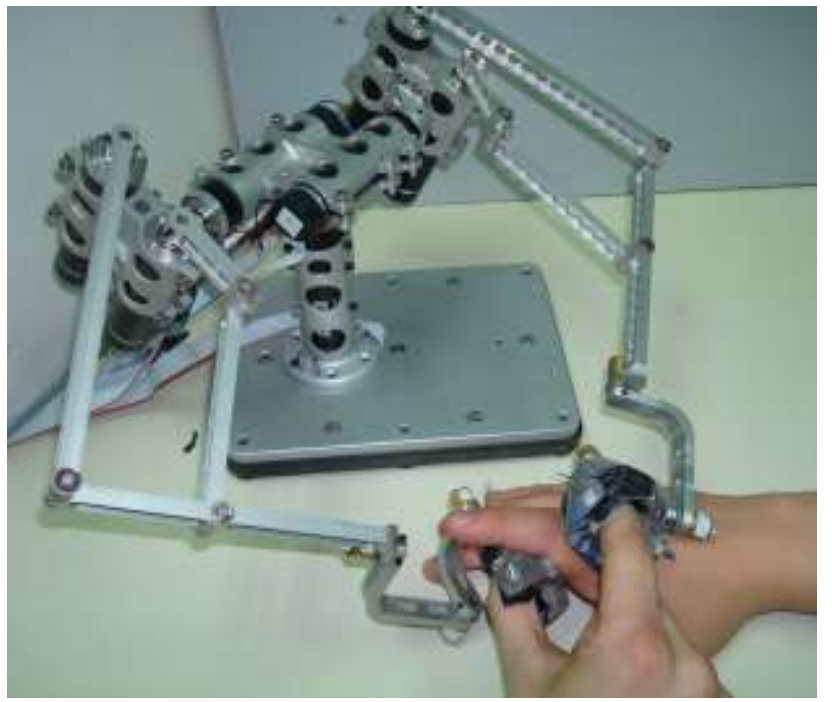

Fig. 4. Capture of forces and movements performing real manipulation in a subject

objects are used to calculate the force.

\section{A. Modeling of the MCP joint}

For this simulation, two different actors have to be modeled: physiotherapist and patient. Physiotherapist actions are managed by the MF-2; that is, the operator inserts their index finger and thumb in the corresponding thimbles to control two blue spheres on the visual display that represent the distal phalange of his/her fingers. Therefore, positions $(x, y, z)$ and orientations $(\alpha, \beta$ and $\gamma)$ of the spheres are controlled by the operator using the MF-2 device.

The model of a patient's hand is divided into two components: the skeleton consisting of a 24 DoF skeleton and the hand shape that includes the skin covering the skeleton [33], as it shown in Fig. 5. Bones are represented by octahedrons and the joints between bones have been defined in Physx. Physx provides different types of joints (fixed, prismatic, spherical, etc.). Simple 1 or 2 DoF joints are usually implemented for other graphical applications, but in order to produce a realistic simulation more DoF are required. Joints between bones are represented by the " 6 DoF Joint" type. The 6 DoF joint type is configured in three linear DoF along the $\mathrm{X}, \mathrm{Y}$ and $\mathrm{Z}$ axis and three angular DoF around the mentioned axis where each DoF is locked, limited or free. Moreover, damping, spring and restitution values for each joint can be defined. The main advantage of using this type of joint and not a specific joint (a spherical joint type in Physx for example could be used to represent the shoulder) is that different limits in each angular and lineal movements can be defined which is not possible when using a spherical joint. In this case, the MCP joint is defined in the following way: the translational movements have been defined as 'locked while the rotations movements have been defined as 'limited'. The angular movements of the joint are defined by three angles: $\alpha$-angle that represents flexion/extension rotations about the $\mathrm{X}$-axis, $\beta$-angle that represents the adduction/abduction (rotation about the $\mathrm{Y}$-axis); and $\gamma$-angle that represents the finger pronation/supination (rotation about the Z-axis).
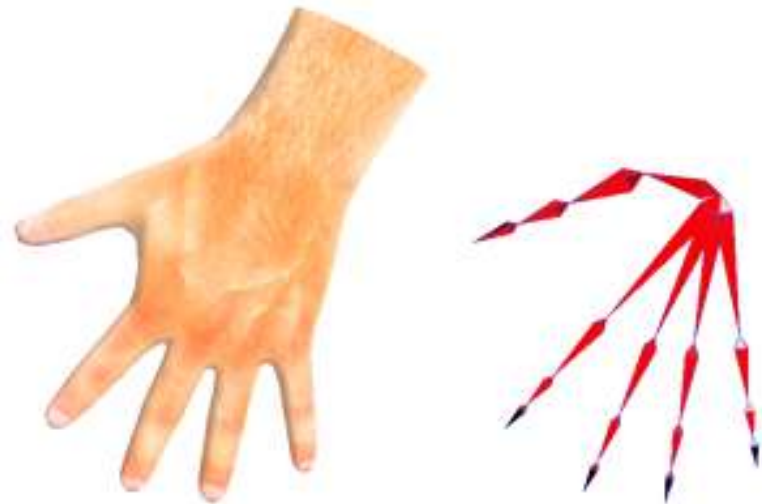

Fig. 5. The shape hand (left) and the 24-DOF skeleton (right).

Once the skeleton and the joints are defined, the shape needs to be modeled. The shapes of the skeleton and skin were modeled using triangle meshes.. As the default collision detection is less robust using triangle meshes, it is necessary to enable the CCD (Continuous Collision Detection) method. As the skin can be deformable, it is also necessary to define a soft body under the skin. Finally, the visual aspect was obtained by scanning a real hand.

\section{B. Characterization and simulation of the MCP joint manipulation}

Two applications are performed using the haptic setup to simulate an assessment and treatment procedure. The assessment consists of an exploration to determine both the quality and quantity of joint movement. The treatment simulation imitates a treatment intended to restore joint function. The maximum force used in producing the reference data was approximately $16 \mathrm{~N}$ and the maximum force that can be produced by the MF-2 is $4 \mathrm{~N}$ so the reflective forces produced were scaled down by a factor of $1: 4$.

The joint movement exploration consisted of checking the MCP joint range and rigidity as would be used in a patient to detect unusual boundaries of the movement. In this exploration the MF-2 directly controls the proximal phalanx of the patient's index finger. The operator starts moving the joint and can both feel and see the resulting movement on the display. The application records the angles that are achieved in each direction. This exploration simulates the operator identifying boundaries and establishing a baseline to evaluate the effectiveness of 
treatment. After the session, the operator can repeat the exploration to reassess the boundaries. Future developments can include changes in the force reflection characteristics over time such as improvement following treatment and transient changes from muscle contraction or protective spasm.

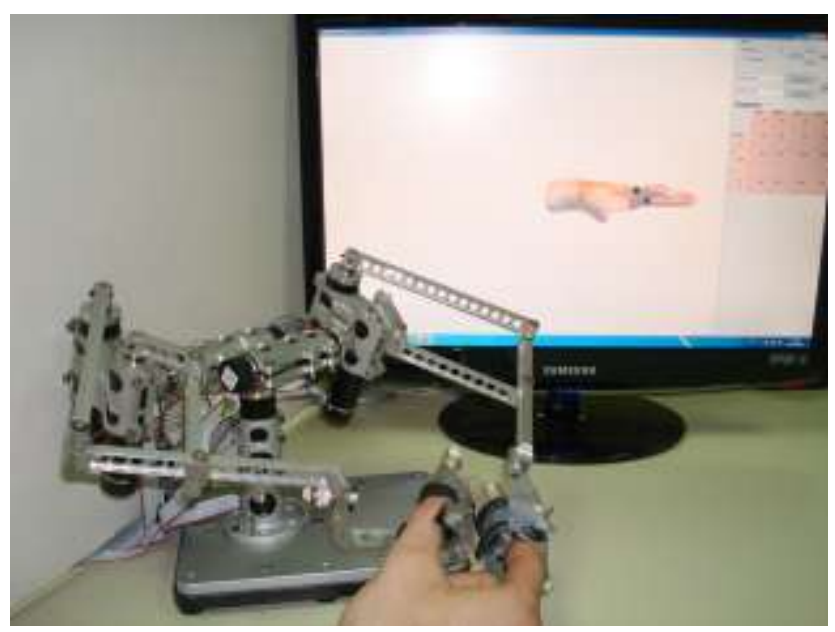

Fig. 6. Application developed for hand manipulation. This application integrates the two-finger haptic device with a human hand virtual model in order to reflect forces that appear during the hand manipulation.

Force treatment simulation consists of exerting an oscillatory force with the thumb and the index finger near the boundaries of the MCP joint motion of the virtual hand in order to simulate the forces needed for the treatment. Physx is only capable of modeling linear stiffness, however as seen in the previous section, the forces required depend of a nonlinear formula. The forces were calculated by other programs based on the equations 1-3 obtained in the previous section. The calculated forces were sent to the MF-2 in order to exert the correct force to the user and to Physx to update the visual information of the virtual model. Moreover, forces are directly related to the angle of the $\mathrm{MCP}$ joint and the penetration of the sphere in the virtual index finger.

The treatment simulation has two parts; first part, the user sees a saved visualization of how the treatment should be performed. He/she sees an animation of a virtual treatment where spheres representing movement of the operator's fingers demonstrate how the movement should be performed and display the force that would be applied to produce this movement. In the second part (Fig. 6), the user directly controls spheres with the MF-2 in an attempt to repeat the same movements and with the same force (magnitude and frequency of the oscillations). The force treatment simulation may be useful for students learning to perform techniques.

\section{CONCLUSIONS}

This paper describes the application of a two-finger haptic device for hand rehabilitation manipulations.
Manipulations, such as exploration and force treatment in which two fingers are required, have been recorded by using the MasterFinger-2 haptic device that has position and contact force sensors, which are used for recording and simulating the hand manipulations. Data can be captured during assessment or treatment of soft tissue, but these tissues cannot be modeled accurately from this limited information. If it is assumed that the bones being manipulated are rigid and have a consistent centre of rotation, the data captured from a series of simple manipulations can be used to produce a functional haptic model

The non-linear properties of the index MCP joint have been estimated by using two thimbles with force sensing resistors as end effectors of a two-finger haptic device. Three axes of movement are needed to model the non-linear pattern of force reflection in order to produce a relatively realistic simulation. The proposed method can be extended to capture rehabilitation techniques and properties of other areas of the human body.

The fact that a preliminary simulation has been possible even with scaled forces being reflected by the haptic device suggests that haptic devices may still be effective teaching tools even if they are not capable of producing the magnitudes of forces applied during real rehabilitation tasks. In rehabilitation manipulation, displacements as well as rotations can be used when applying treatments, so future applications may need to include three linear DoFs as well as three rotational DoFs used in this application.

The potential application of this work is related to medical and educational purposes. Students typically learn their skills in manipulating musculoskeletal structures by practicing on their fellow students or, under supervision, on a limited number of patients. With current methods, it is difficult for students to receive objective feedback and is also unlikely that they will have the opportunity to palpate even the range of the most of the common conditions that are presented by patients.

Practice with virtual models therefore could not only improve the efficiency of early skill development through objective feedback, but also broaden the experience of students to enable them to recognize a wider range of presentations including rare conditions that they would be unlikely to encounter through the current training methods. Another potential application of these results is the assessment of students that are not in campus; the students can send videos and forces recorded while performing the techniques for evaluations. This process is not unreasonable as currently some universities use video without force data in teaching and assessment when students are at distance (not in campus). Future works of the presented results will include extension of these principles to develop palpable virtual reality models of other joints and areas of the body, evaluation of student's performance, and evaluation of improvements achieved in teaching. 


\section{REFERENCES}

[1] M. C. Harms and D. L. Bader, "Variability of forces applied by experienced therapists during spinal mobilization," Clinical Biomechanics, vol. 12, Sep 1997, pp. 393-399.

[2] J. Marcotte, M.C. Normand, and P. Black, "Measurement of the pressure applied during motion palpation and reliability for cervical spine rotation," Journal of Manipulative and Physiological Therapeutics, vol. 28, pp. 591-6, Oct 2005.

[3] S.J. Snodgrass, D.A. Rivett, and V.J. Robertson, "Manual forces applied during posterior-to-anterior spinal mobilization: a review of the evidence," Journal of Manipulative and Physiological Therapeutics, vol. 29, pp. 316-29, May 2006.

[4] S. J. Snodgrass, et al., "Forces applied to the cervical spine during posteroanterior mobilization," J Manipulative Physiol Ther, vol. 32, pp. 72-83, Jan 2009.

[5] D. Shirley, "Manual therapy and tissue stiffness," in Grieve's Modern Manual Therapy, J. D. Boyling and G. Jull, Eds., Third ed Sydney: Churchill Livingstone, 2004, pp. 381-391.

[6] L. Nicholson, C. Maher, R. Adams, and N. Phan-Thien, "Stiffness properties of the human lumbar spine: a lumped parameter model," Clinical Biomechanics, vol. 16, pp. 285-92, May 2001.

[7] N. Tuttle, R. Barrett, and L. Laakso, "Relation between changes in posteroanterior stiffness and active range of movement of the cervical spine following manual therapy treatment," Spine, vol. 33, pp. E673-9, Sep 12008.

[8] C.G. Maher, M. Simmonds, and R. Adams, "Therapists' conceptualization and characterization of the clinical concept of spinal stiffness," Physical Therapy, vol. 78, pp. 289-300, Mar 1998.

[9] Sha Ma; Varley, M.R.; Richards, J.; Lik-Kwan Shark; , "Overcoming the Information Overload Problem in a Multiform Feedback Based Virtual Reality System for Hand Motion Rehabilitation," CyberWorlds, 2009. CW '09. International Conference on, vol., no., pp.51-56, 7-11 Sept. 2009

[10] Maciel A, Halic T, Lu Z, Nedel LP, De S. Using the PhysX engine for physics-based virtual surgery with force feedback. Int J Med Robot. 2009 Sep;5(3):341-53.

[11] Zahraee, A.H.; Paik, J.K.; Szewczyk, J.; Morel, G.; , "Toward the Development of a Hand-Held Surgical Robot for Laparoscopy," Mechatronics, IEEE/ASME Transactions on , vol.15, no.6, pp.853861, Dec. 2010

[12] Mali, U. and Munih, M., "HIFE-Haptic Interface for Finger Exercise" in IEEE Transactions on Mechatronics, Vol. 11, No.1, Febrary 2006.pp. 93-102

[13] Blake, J. and Gurocak, "Haptic Globe with MR Brakes for Virtual Reality" in IEEE Transactions on Mechatronics, Vol. 14, No.5, October 2009. p.p.606-615

[14] S. V. Adamovich, A. S. Merians, R. Boian, M. Tremaine, G. S. Burdea, M. Recce and H. Poizner, "A Virtual Reality Based Exercise System for Hand Rehabilitation Post-Stroke: Transfer to Function", in Proc. of the 26th Annual International Conference of the IEEE EMBS, San Francisco, CA, USA, 2004, pp. 4936-4939.

[15] S.V. Adamovich, G.G. Fluet, A. Mathai, Q. Qiu, J. Lewis, A.S. Merians. "Design of a complex virtual reality simulation to train finger motion for persons with hemiparesis: A proof of concept study. Journal of Neuroengineering and Rehabilitation". Vol. 6, $\mathrm{n}^{\circ}$ 28, 2009.

[16] Huber, M.; Rabin, B.; Docan, C.; Burdea, G.C.; AbdelBaky, M.; Golomb, M.R.; , "Feasibility of Modified Remotely Monitored InHome Gaming Technology for Improving Hand Function in Adolescents With Cerebral Palsy," Information Technology in Biomedicine, IEEE Transactions on , vol.14, no.2, pp.526-534, March 2010

[17] Ioannis Sarakoglou, Sophia Kousidou, Nikolaos G. Tsagarakis and Darwin G. Caldwell. Exoskeleton-Based Exercisers for the Disabilities of the Upper Arm and Hand, Rehabilitation Robotics, Sashi S Kommu (Ed.), ISBN: 978-3-902613-04-2, p.p. 500-522, 2007.

[18] Shakra, I.; Orozco, M.; Saddik, A.E.; Shirmohammadi, S.; Lemaire, E.; , "Haptic Instrumentation for Physical Rehabilitation of Stroke Patients," Medical Measurement and Applications, 2006. MeMea 2006. IEEE International Workshop on , vol., no., pp.98-102, 20-21 April 2006

[19] Q. Qiu, G. G. Fluet, I. Lafond, A. S. Merians, and S. V. Adamovich, "Coordination changes demonstrated by subjects with hemiparesis performing hand-arm training using the njit- ravr robotically assisted virtual rehabilitation system." Annual International Conference of the IEEE Engineering in Medicine and Biology Society. IEEE Engineering in Medicine and Biology Society. Conference, vol. 2009, pp. 1143-1146, 2009

[20] Adamovich, S.V.; Merians, A.S.; Boian, R.; Tremaine, M.; Burdea, G.S.; Recce, M.; Poizner, H.; , "A virtual reality based exercise system for hand rehabilitation post- stroke: transfer to function," Engineering in Medicine and Biology Society, 2004. IEMBS '04. 26th Annual International Conference of the IEEE, vol.2, no., pp.4936- 4939, 1- 5 Sept. 2004

[21] Ueki, Satoshi; Nishimoto, Yutaka; Abe, Motoyuki; Kawasaki, Haruhisa; Ito, Satoshi; Ishigure, Yasuhiko; Mizumoto, Jun; Ojika, Takeo; , "Development of virtual reality exercise of hand motion assist robot for rehabilitation therapy by patient self- motion control," Engineering in Medicine and Biology Society, 2008. EMBS 2008. 30th Annual International Conference of the IEEE , vol., no., pp.4282- 4285, 20- 25 Aug. 2008

[22] Ueki, S.; Kawasaki, H.; Ito, S.; Nishimoto, Y.; Abe, M.; Aoki, T.; Ishigure, Y.; Ojika, T.; Mouri, T.; , "Development of a Hand-Assist Robot With Multi-Degrees-of-Freedom for Rehabilitation Therapy," Mechatronics, IEEE/ASME Transactions on , vol.PP, no.99, pp.111,2010

[23] Tholey, G.; Desai, J.P.; , "A General-Purpose 7 DOF Haptic Device: Applications Toward Robot-Assisted Surgery," Mechatronics, IEEE/ASME Transactions on, vol.12, no.6, pp.662-669, Dec. 2007

[24] P. García-Robledo, J. Ortego, M. Ferre, J. Barrio, and M.A. Sánchez-Urán, "Segmentation of Bimanual Virtual Object Manipulation Tasks Using Multifinger Haptic Interfaces", in IEEE Trans. on Instrumentation and Measurement. Vol. 60 , Issue 1, Jan 2011. pp. 1-12.

[25] Cobos, S.; Ferre, M.; Aracil, R.; , "Simplified human hand models based on grasping analysis," Intelligent Robots and Systems (IROS), 2010 IEEE/RSJ International Conference on , vol., no., pp.610-615, 18-22 Oct. 2010.

[26] M. Monroy, M. Ferre, J. Barrio, V. Eslava and I. Galiana. "Sensorized thimble for haptics applications". Proceedings of the 2009 IEEE International Conference on Mechatronics. Málaga,Spain. April 2009. pp 1-6.

[27] Garcia-Robledo, P.; Ortego, J.; Barrio, J.; Galiana, I.; Ferre, M.; Aracil, R.; , "Multifinger haptic interface for bimanual manipulation of virtual objects," Haptic Audio visual Environments and Games, 2009. HAVE 2009. IEEE International Workshop on , vol., no., pp.30-35, 7-8 Nov. 2009.

[28] Galiana, I., Bielza, M. and Ferre, M. "Estimation of Normal and Tangential Manipulation Forces by using Contact Force Sensors" In Proceedings of Eurohaptics 2010, Amsterdam, The Netherlands. pp 65-72

[29] Tekscan. (2009, January 15, 2010). Flexiforce Sensors Manual. Available:

http://www.tekscan.com/pdfs/FlexiforceUserManual.pdfhttp://www. tekscan.com/pdfs/FlexiforceUserManual.pdf

[30] N N.R. Crawford, J.D. Peles, and C.A. Dickman, "The spinal lax zone and neutral zone: measurement techniques and parameter comparisons," Journal of Spinal Disorders, vol. 11, pp. 416-29, Oct 1998.

[31] M. Lee, J. Gal, and W. Herzog, "Biomechanics of manual therapy," in Clinical Biomechanics, Z. Dvir, Ed., ed Philadelphia: Churchill Livingstone, 2000, pp. 209-237.

[32] D. Werner, S.H. Kozin, M. Brozovich, S.T. Porter, D. Junkin, and S. Seigler, "The biomechanical properties of the finger metacarpophalangeal joints to varus and valgus stress," J Hand Surg Am, vol. 28, pp. 1044-51, Nov 2003.

[33] S. Cobos, M. Ferre, M.A. Sanchez-Uran, J. Ortego, R. Aracil, "Human hand descriptions and gesture recognition for object manipulation", in Computer Methods in Biomechanics and Biomedical Engineering Vol. 13, No. 3, June 2010, pp: 1476-1482.

[34] N. Tuttle and G. Jacuinde, "Design and construction of a novel lowcost device to provide feedback on manually applied forces," J Orthop Sports Phys Ther, vol. 41, pp. 174-9, Mar 2011. 


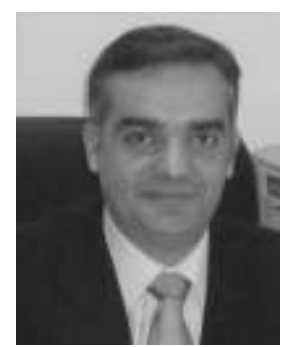

Manuel Ferre (M'00) received the Laurea degree in Control Engineering and Electronics and the Ph.D. degree in Automation and Robotics from the Universidad Politécnica de Madrid (UPM), Madrid, Spain, in 1992 and 1997, respectively.

In 1990, he started his research activity at UPM, where he has been a Professor Titular since 2000. In 1997, he worked as a PostDoc in the Human-Machine System Laboratory, Massachusetts Institute of Technology (MIT), Cambridge. He has participated and coordinated several research projects in robotics and automatic control, both at national and international programs. His research interests are focused on automatic control, advanced telerobotics, and haptics. He has two patents of haptic devices and stereoscopic video cameras. He has been a Coeditor of Advances in Telerobotics, a book of Springer STAR series and author of many papers on bilateral control and human interfaces for teleoperation.

Dr. Ferre serves as Chair of the Robotics \& Automation Society Technical Committee on Telerobotics. He is also a member of EuroHaptics Society where he serves as Treasurer. He has participated in International Program Committees of several conferences highlighting some editions of Intelligent Robots and Systems and EuroHaptics. He was the Chairman of the First International Workshop on Telerobotics in 2004 and hosted the EuroHaptics 2008 conference.

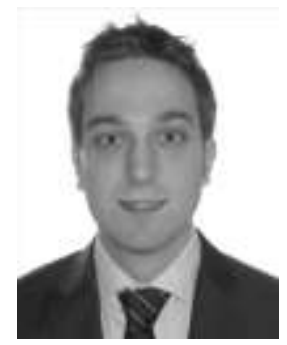

Ignacio Galiana was born in La Rioja, Spain in 1986. He received a 5-year B.Sc. degree in Industrial Engineering with a major in Automation and Electronics from the Universidad Politécnica de Madrid, Spain, in 2009, and a M.Sc. degree (with honors) in automation and control engineering from the Universidad Politécnica de Madrid, Spain, in 2010.

In 2009, he joined the Centre for Automation and Robotics, UPM-CSIC, as a researcher where he has been awarded a grant to work on his Ph.D. degree. He has participated in research projects in robotics and control, both at national and international programs.

His current research focuses on bilateral Control, biomedical applications, force sensing technologies and applications of teleoperation Systems. Mr. Galiana is student member of the the Robotics \& Automation IEEE Society, member of EuroHaptics Society and IEEE-RAS Technical Committee on Telerobotics.

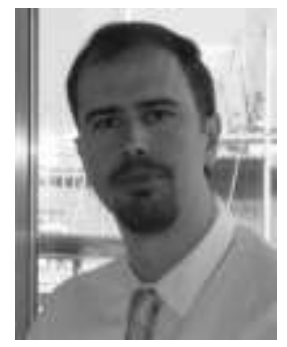

Raúl Wirz received the M.S. degree in Computer Science from Jaume I University (UJI), Castellon, Spain, in 2004, and the Ph.D. degree in Computer Science from Jaume I University (UJI), Castellon, Spain, in 2009. His $\mathrm{Ph} . \mathrm{D}$. dissertation involves networked robots. In 2003, he joined the Robotic Intelligence Laboratory, Department of Computer Science, UJI, and worked on research projects related to Internet telerobotics, Internet transport protocols and human-robot interfaces.

In 2009, he joined the Centre of Automation and Robotics, UPM-CSIC, and worked on research projects related to virtual environments (PhysX and DirectX) and haptic devices for rehabilitation tasks. His current research interests include haptic devices, rehabilitation Technology and 3D virtual environments.

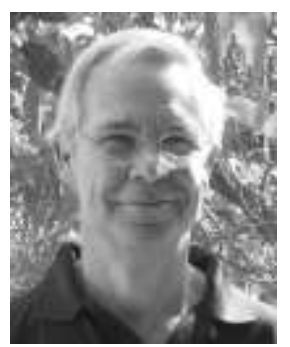

Neil Tuttle received a BSc. degree in Physical Therapy from the University of Michigan, Ann Arbor, in 1973, a Graduate Diploma in Advanced Manipulative Therapy from South Australian Institute of Technology, Adelaide, Australia, in 1976, and an MPhil in 2000 and Ph.D. in 2008, both in Physiotherapy and from Griffith University, Queensland, Australia.

For the past 35 years he has combined teaching and clinical practice in Physiotherapy and in the past ten years has been more extensively involved in research. He continues in private clinical practice and is currently a Senior Lecturer in Physiotherapy at Griffith University in Australia with a particular interest in understanding and teaching of manual therapy skills and clinical reasoning. 